\title{
Advances in Pathogenesis of Idiopathic Membranous Nephropathy
}

\author{
Zhifeng $\mathrm{Xu}^{\mathrm{a}}$ Lu Chen ${ }^{\mathrm{b}}$ Huiling Xiang ${ }^{\mathrm{a}}$ Chun Zhang $^{\mathrm{a}}$ Jing Xiong ${ }^{\mathrm{a}}$ \\ aDepartment of Nephrology, Union Hospital, Tongji Medical College, Huazhong University of Science and \\ Technology, Wuhan, China; ${ }^{b}$ Department of Geriatrics, Union Hospital, Tongji Medical College, Huazhong University \\ of Science and Technology, Wuhan, China
}

\section{Keywords}

Membranous nephropathy · Phospholipase A2 receptor .

Thrombospondin type-1 domain-containing 7A. Podocyte autoantigen $\cdot$ Single nucleotide polymorphisms

\section{Abstract}

Background: Membranous nephropathy (MN), a major cause of nephrotic syndrome, has attracted people's attention in recent years for its growing prevalence. It is the second or third leading cause of ESRD in patients with primary glomerulonephritis and is the leading glomerulopathy that recurs after kidney transplantation. Summary: MN can be classified as idiopathic membranous nephropathy (IMN) and secondary MN. The discovery of the M-type phospholipase $\mathrm{A} 2$ receptor (PLA2R) and thrombospondin type-1 domain-containing 7A (THSD7A) provides the new diagnostic methods and treatment strategies for IMN on the molecular level. The study on single nucleotide polymorphism of IMN genes, such as the single $\mathrm{M}$-type phospholipase $\mathrm{A} 2$ receptor 1 (PLA2R1) gene and human leukocyte antigen (HLA) gene, explains the pathogenesis of the disease from the perspective of genetics and conforms to the trend of the era of precision medicine. Key Messages: This review focuses on advances in the pathogenesis of IMN, including molecular and

\begin{tabular}{ll}
\hline karger@karger.com & ( ) 2020 The Author(s) Karger \\
www.karger.com/kdd & Published by S. Karger AG, Basel Open access \\
& This article is licensed under the Creative Commons Attribution- \\
Narger & Nommercial-NoDerivatives 4.0 International License (CC BY- \\
NC-ND) (http://www.karger.com/Services/OpenAccessLicense). \\
Usage and distribution for commercial purposes as well as any dis- \\
tribution of modified material requires written permission.
\end{tabular}

genetic pathogenesis, as well as discussing the diagnostic and treatment guiding value brought by these new discoveries.

(C) 2020 The Author(s)

Published by S. Karger AG, Basel

\section{Introduction to Membranous Nephropathy}

Membranous nephropathy ( $\mathrm{MN})$ is a major cause of nephrotic syndrome in adults with nondiabetic origin. It is the second or third leading cause of ESRD in patients with primary glomerulonephritis and is the leading glomerulopathy that recurs after kidney transplantation [1]. MN can occur in people of all ages, but is more common in general adults. The typical clinical manifestations of MN are edema, massive proteinuria, hypoalbuminemia, and hyperlipidemia. Its characteristic pathological manifestations include diffuse homogeneous thickening of the glomerular basement membrane (GBM) and the formation of spike under a light microscope; granular deposition of immunoglobulin G (IgG), and $\mathrm{C} 3$ in glomerular capillary loops under immunofluorescence; extensive fusion of foot processes of podo-

Zhifeng Xu and Lu Chen have contributed equally to this work.

Jing Xiong

Department of Nephrology, Union Hospital, Tongji Medical College Huazhong University of Science and Technology

1277 Jiefang Avenue, Wuhan 430022 (China)

jingxiong@ hust.edu.cn 
cytes; and subepithelial electron dense deposits under an electron microscope.

According to the etiology, MN can be classified as idiopathic membranous nephropathy (IMN) and secondary membranous nephropathy (SMN). Factors currently reported that can cause SMN [2] mainly include autoimmune diseases (systemic lupus erythematosus [SLE], rheumatoid arthritis, Sjögren's syndrome, mixed connective tissue disease, and thyroiditis), tumors (thyroid tumors, solid tumors such as lung cancer and colon cancer, leukemia, lymphoma, and other malignant tumors of the blood), chronic infection (hepatitis B and C, syphilis, malaria, and leprosy), drugs and poisons (nonsteroidal antiinflammatory drugs, gold agents, penicillamine, captopril, and heavy metals), allogeneic hematopoietic stem cell transplantation, and graft versus host disease. These secondary factors should be excluded before the diagnosis of IMN. So far, most scholars believe that IMN is an autoimmune disease mediated by antigen-antibody complexes; the antigens include neutral endopeptidase (NEP), M-type phospholipase A2 receptor (PLA2R), and thrombospondin type-1 domain-containing 7A (THSD7A), and other endogenous podocyte antigens binding to autoantibodies, forming subepithelial deposits in the lateral basement membrane, activating the complement system, which cause damage to podocytes and lead to proteinuria [1]. It has also been reported that environmental pollution and genetic factors are closely related to the occurrence of IMN [3].

In the natural course of IMN, about $1 / 3$ IMN patients can receive spontaneous remission and other $1 / 3$ can achieve remission after immunosuppressive treatment, while the rest of them will progress to renal failure with uncontrolled proteinuria and repeated attacks. Because of the high incidence and recurrence of the disease, it is of importance to research IMN pathogenesis for effective treatment, prognosis evaluation, and treatment adjustment. Here, this article focuses on IMN molecular and genetic level pathogenesis, revealing the correlation between IMN molecular markers and clinical practice.

\section{Research Progress of Pathogenesis on Molecular Level}

\section{Autoantigen of Podocytes}

M Phospholipase A2 Receptor

In 2009, Beck et al. [4] detected a glycoprotein molecule with a size of $185 \mathrm{kDa}$ from the glomerular extract of IMN patients by Western blot (WB), which could be im- munoprecipitated with the serum components of IMN patients. They identified the molecule as PLA2R by mass spectrometry analysis. This receptor, a transmembrane protein, expressed in normal podocytes, can also be seen in the glomerular immune complex of IMN patients. Anti-PLA2R antibodies can be eluted from renal biopsy tissues of IMN patients, and antibodies against PLA2R conformation-dependent epitopes are present in the serum of about $70 \%$ of patients. The antibody is mainly IgG4 subtype, co-localized with PLA2R in the glomeruli. However, Beck et al. [4] did not detect the presence of specific IgG4 antibody against PLA2R in the serum of patients with membranous lupus nephritis and IgA nephropathy. Recently, von Haxthausen et al. [5] found that PLA2R antigen appeared in the bronchial epithelial cells in IMN patients with positive anti-PLA2R antibody. But lung damage is seldom found in $\mathrm{MN}$ patients; the reason may be the physiological and anatomical structure differences between bronchioles and glomerular epithelial cells. Bronchial epithelial cells in an interconnected environment, with a faster cellular renewal rate, and its immune deposits can be quickly removed by sputum. However, the immune complex deposited under the glomerular epithelium is difficult to be removed, which leads to kidney damage.

PLA2R is a type I transmembrane receptor, belonging to one of the four mannose receptor families with conserved domains. It consists of a large extracellular segment, a transmembrane domain, and a short intracellular tail segment. The extracellular segment contains the $\mathrm{N}$ terminal cysteine-rich ricin domain (CysR), fibronectin type II domain (FnII), and eight C-type lectin domains (CTLDs) [6]. Kao et al. [7] found that a major antigen epitope of PLA2R was a complex composed of N-terminal CysR-FNII-CTLD1, and any deficiency in CysR or CTLD1 would prevent specific antibodies from recognizing the remaining regions in PLA2R molecules. Because the complex contains at least one disulfide bond, it almost completely blocks the reactivation of the autoantibody to the entire PLA2R. Shortly after, CysR was narrowed as a major antigen epitope of PLA2R by Fresquet et al. [8], where they identified a 31-amino acid sequence that can be recognized by $90 \%$ of anti-PLA2R antibodies. Through antibody blocking and circulating autoantibody immunoadsorption, it is expected to block the occurrence of the disease from the source.

How is the immune response of IMN induced? There are several hypotheses. First, PLA2R-associated peptides released by podocytes or other cells bind specifically to the human leukocyte antigen (HLA)-DOA1 molecule's 
antigen-binding site, triggering an immune response. Second, molecular simulation of PLA2R epitopes by microorganisms or other environmental antigens enables genetically susceptible populations with HLA-DOA1 variants to produce anti-PLA2R antibodies, inducing an immune response [8]. Third, circulating anti-PLA2R antibodies and podocyte PLA2R antigens in IMN patients are deposited in situ in the GBM, activating the complement system and mediating immune damage. At present, the third hypothesis is highly accepted. In addition, expansion of B-cell epitopes or regulatory T cells may also be involved in the pathogenesis of IMN, but the specific mechanism remains to be verified by subsequent studies [7-9].

\section{PLA2R1 Epitope Spreading}

In general, epitope spreading produces a more robust immune response to a given antigen. Although this is desirable to fight microbial invasion and cancer spread, it may make an autoimmune condition more resistant to the restoration of self-tolerance and spontaneous remission or drug-induced immune suppression. Such seems to be the case in PLA2R-associated MN [7]. Seitz-Polski et al. [10] screened 69 patients with IMN disease and identified epitopes in three domains of PLA2R1: CysR, CTLD1, and CTLD7. In their study, patients with antiCysR-restricted activity were younger, had less nephrotic range proteinuria, and exhibited a higher rate of spontaneous remission and lower rates of renal failure progression during follow-up. Anti-CTLD1 and anti-CTLD7 antibodies disappeared with disease remission and reappeared with disease relapse, while anti-CysR-restricted activity was associated with stable and mild disease activity, suggesting that a second immune challenge (allergy, infection, etc.) might induce epitope spreading from

Fig. 1. Mechanisms of kidney injury in MN. a The peptides of PLA2R released by podocytes or other cells, and molecular simulation of PLA2R epitopes by microorganisms or other environmental antigens, triggering immune response, enable lymphocytes to produce anti-PLA2R antibodies to attack PLA2R in the kidney. The extracellular segment of the phospholipase A2 receptor (PLA2R) contains an N-terminal CysR region, an FnIID, and a tandem repeat of eight CTLDs. The major antigen epitope of PLA2R is in CysR which can be bound by anti-PLA2R antibodies. A second immune challenge (allergy, infection, etc.) induces epitope spreading from CysR toward CTLD1 and CTLD7, which induces more anti-PLA2R antibodies and exacerbates renal injury. b The immune system recognizes the endogenous integral membrane proteins of the podocyte or exogenous antigens planted in the glomerulus and produces antibodies that attack the kidney.
CysR toward CTLD1 and CTLD7, which induces more anti-PLA2R antibodies and exacerbates renal injury (Fig. 1a). In their prospective cohort study [11], 58 patients positive for anti-PLA2R1 antibody were randomly allocated to rituximab or anti-proteinuric therapy alone. They analyzed the predictive value of anti-PLA2R1 antibody titers and epitope spreading. The result showed that the epitope spreading was strongly correlated with antiPLA2R1 antibody titer. Ten of the 17 (58.8\%) patients who had epitope spreading at baseline and were treated with rituximab showed reversal of epitope spreading at the 6th month. In adjusted analysis, epitope spreading at baseline was associated with a decreased remission rate at the 6th month and last follow-up, independently from age, sex, baseline anti-PLA2R1 antibody level, and treatment group. Their data suggest that a patient who has only a low level of anti-PLA2R1 antibody but significant spreading should be treated with rituximab at the time of diagnosis. A patient who has high anti-PLA2R1 antibody levels but no spreading could be observed without treatment. However, these potentially important results need to be confirmed in further studies.

\section{Thrombospondin Type-1 Domain-Containing 7A}

Are there other specific antigens related to IMN besides PLA2R? In 2014, Tomas et al.[12] isolated and purified a new antigen THSD7A by using a method similar to the previous PLA2R antigen search. This antigen, mainly co-localized with IgG4 in the subepithelial space, has similar structural and biochemical properties to PLA2R and is present in both glomerular podocytes of the normal population and glomerular immune complexes of IMN patients. They also found that antibodies against this antigen were detected in 15 of the 154 (about 10\%) IMN patients' serum, and subsequent studies also showed that

c Complement can be activated by three different pathways in IMN. The C5b-9 membrane attack complex is formed after the activation of complement, which induces the production of ROS, leading to GBM damage and proteinuria, and causes apoptosis and exfoliation of podocytes directly, leading to a reduced number of glomerular podocytes. PLA2R, phospholipase A2 receptor; THSD7A, thrombospondin type-1 domain-containing 7A; CysR, cysteinerich ricin domain; FnIID, fibronectin-like type II domain; CTLD, C-type lectin-like domain; NEP, neutral endopeptidase; BSA, bovine serum albumin; ROS, reactive oxygen species; GBM, glomerular basement membrane; SOD2, superoxide dismutase 2; AR, aldose reductase; NELL-1, neural epidermal growth factor-like 1 protein; GBM, glomerular basement membrane; Ig, immunoglobulin; MBL, mannose-binding lectin; MASP, mannan-binding lectin-associated serine protease; $\mathrm{MN}$, membranous nephropathy.

(For figure see next page.) 


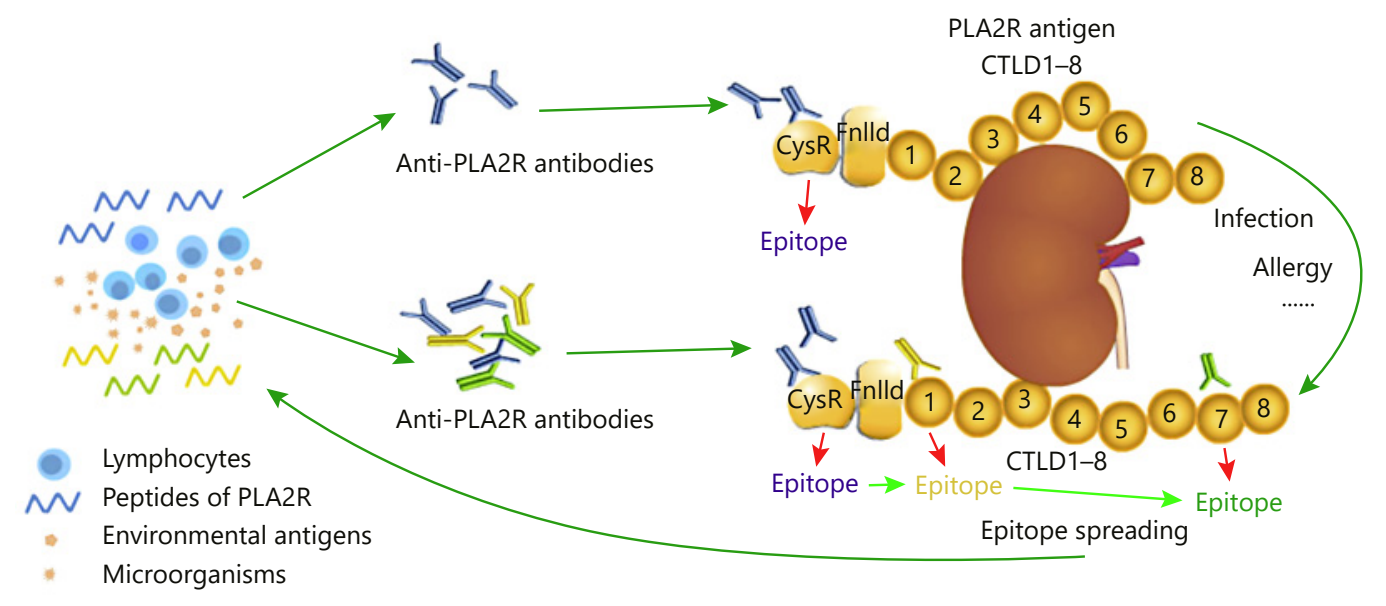

a

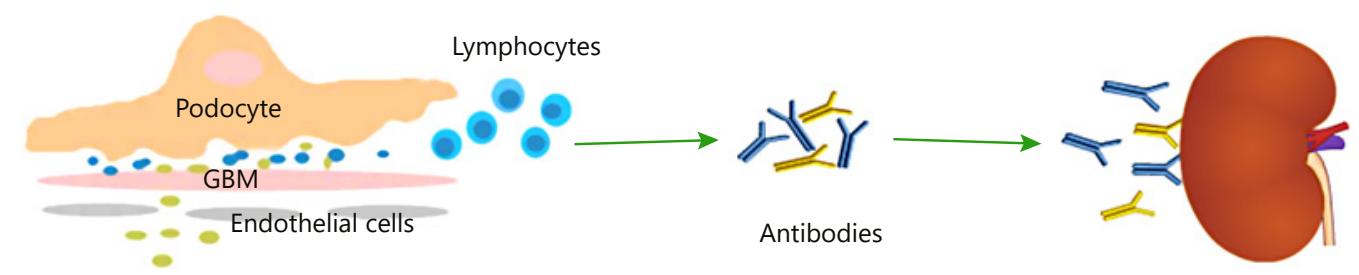

- Autoantigen of podocytes (PLA2R, THSD7A, NEP, AR, SOD2 or NELL-1)

b

- Planting antigen (Cationic BSA)
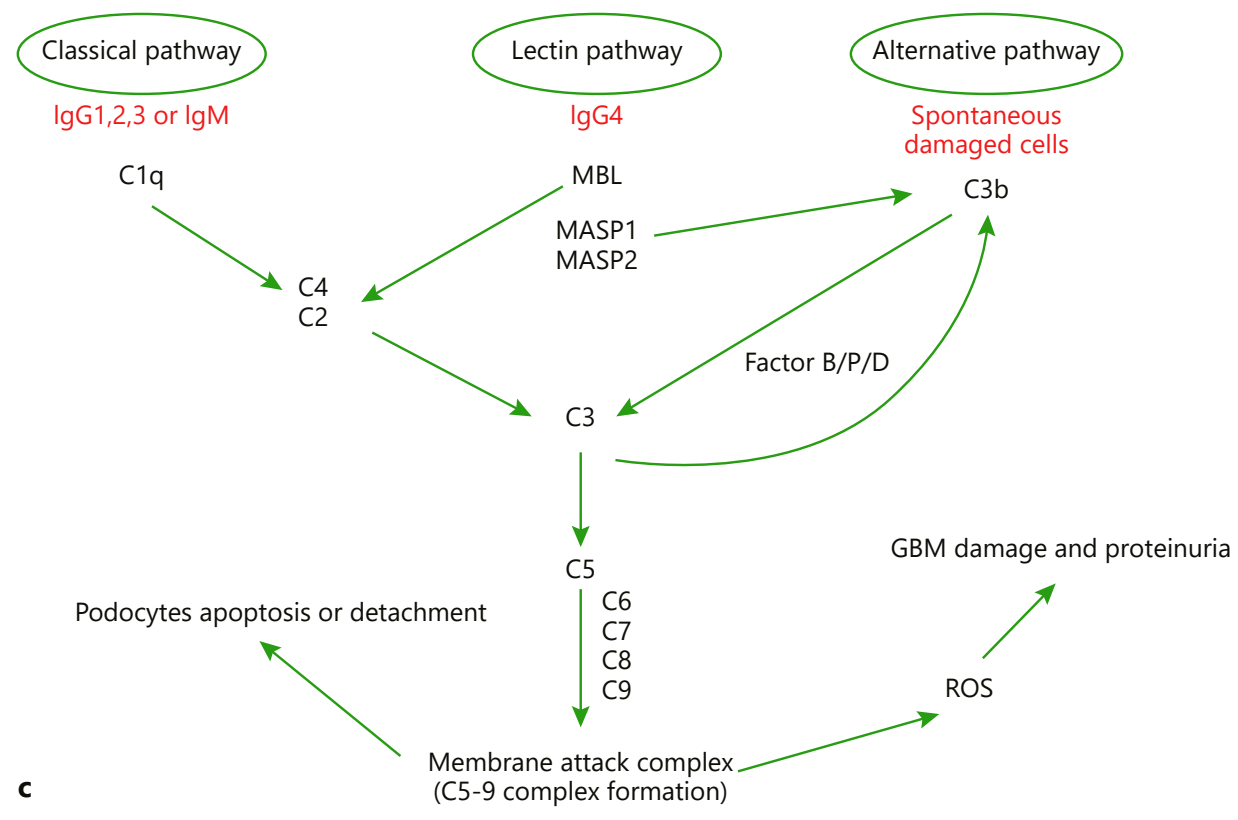

1 
circulating anti-THSD7A antibodies were present in about $5-10 \%$ of patients with negative anti-PLA2R antibodies. This finding indicates that a few IMNs can be mediated by THSD7A antigen and its specific antibody. According to Salant's review, THSD7A was originally identified in placental vessels and human umbilical vein endothelial cells, co-localizing with $\alpha \mathrm{V} \beta 3$ integrin and paxillin, linking to the actin cytoskeleton, and regulating endothelial migration. It was also demonstrated in the neural tube of developing zebrafish embryos along the growth path of angiogenic intersegmental vessels and in intestinal vessels, but it was not expressed in glomerular endothelial cells. Nonetheless, the ability of thrombospondin type 1 repeats to engage in cell-cell and cellmatrix interactions, as well as the studies with THSD7A in human umbilical vein endothelial cells and zebrafish, suggests that THSD7A may contribute to podocytes' adhesion to the GBM. Moreover, it is possible that proteolytic cleavage and release of soluble THSD7A during antibody-mediated injury may influence such adhesion and, thereby, cause effacement of the podocyte foot process [13].

However, recent studies have shown that THSD7A is closely in correlation with MN related to tumor. Hoxha et al. [14] reported that THSD7A was detected in the cancer tissues and metastatic lymph nodes of a patient with gallbladder cancer. After chemotherapy, the titer of serum anti-THSD7A antibody significantly decreased and urine protein apparently improved. They also screened $1,009 \mathrm{MN}$ patients and found 25 of them with circulating anti-THSD7 A antibodies, including 7 with malignant tumors. Hara et al. [15] found that among 469 consecutive cases of pathologically confirmed MN diagnosed at four centers in Japan, 14 cases were confirmed positive for THSD7A (3.0\%). Cancer was detected at the time of renal biopsy (small-cell carcinoma of the lung and prostatic adenocarcinoma with neuroendocrine differentiation) in 2 patients, and both tumors were negative for THSD7A. Four patients had concurrent or previous incidence of allergic diseases, including 1 patient with Kimura's disease. All these findings indicated that THSD7A may have a possible association not only with tumors but also with allergic disorders.

Zaghrini et al. [16] screened 1,012 MN patients and found a total of 49 THSD7A-related patients. Further study found that the age of female patients was significantly lower than that of male patients, suggesting that the etiology of this antigen may be different from other pathogenic factors. Follow-up of 12 patients showed that the anti-THSD7A antibody titer significantly decreased or disappeared in patients who received spontaneous remission and remission after immunosuppressive treatment, while patients whose antibody titer remained at a high concentration could not receive remission, and also the remission rate in patients with low antibody titer was higher than that in those with high titer, suggesting the relationship between anti-THSD7A antibody and disease activity. Moreover, the relationship between THSD7A and IMN remains to be further verified.

\section{Neutral Endopeptidase}

Since 2002, NEP antigens in glomerular podocytes have attracted more and more attention. This year, Debiec et al. [17] reported an infant with severe congenital $\mathrm{MN}$, which may be caused by anti-NEP antibodies from the mother. These antibodies were found in the infant's serum 13 days after birth, but then disappeared, indicating passive transplacental immunization. NEP deficiency caused the alloimmunization in the mother that most likely occurred at the time of her miscarriage. During the subsequent pregnancy, NEP antibodies from the mother can pass through the placenta and bind to the NEP on fetal podocytes, leading to maternal-fetal alloimmune $\mathrm{MN}$. Over the next few years, familial clustering of this kind of $\mathrm{MN}$ was found. The circulating anti-NEP antibody titers of seven mothers from five families were high, and their children were affected. Due to the same truncated mutation in exon 7 of the membrane metallo-endopeptidase (MME) gene, all immunized mothers were NEP deficient. The mother of the first family is a compound heterozygote of the mutation and another truncated mutation of exon $15[18,19]$. With the decrease in maternal antibody titer after birth, the renal function of most newborns returned to normal, but a few of them eventually developed into CKD with nephron deficiency. Therefore, early gene screening for mothers with a history of disease and their family members is essential to prevent CKD caused by nephron deficiency [18].

\section{Other Autoantigens}

No evidence of PLA2R and THSD7A antigens has been found in about $10-20 \%$ of IMN, and other self-antigens such as superoxide dismutase 2 (SOD2) and aldose reductase $(\mathrm{AR})$ may play a role in the pathogenesis of IMN. In 2011, Prunotto et al. [20] reported that specific anti-AR and anti-SOD2 IgG4 antibodies could be detected in the serum and the eluent of kidney biopsy immune complex of MN patients, and that these antibodies and c5b-9 were co-located in the podocyte immune complexes. In vitro experiments also showed that the expression 
of SOD2 on the plasma membrane of podocytes was increased after incubation with hydrogen peroxide. Accordingly, Prunotto et al. [20] proposed that AR and SOD2 are also target antigens of $\mathrm{MN}$ in humans, and oxidative stress can stimulate the expression of SOD2 in the glomeruli. A clinical study by Han et al. [21] found that among the $56 \mathrm{IMN}$ patients, 26 were positive for anti-AR antibody and 38 were positive for anti-SR antibody, but the positive rate of anti-AR antibody was not statistically different from that of the control group. Although the antibodies of the two were positively correlated with the level of proteinuria, they had no apparent significance for the progress of $\mathrm{MN}$ and had poor specificity for the diagnosis of $\mathrm{MN}$, so they cannot be used as indicators for the diagnosis and detection of $\mathrm{MN}$ in clinical practice. The relationship between $\mathrm{AR}, \mathrm{SOD} 2$, and IMN needs further research and explorations.

A novel autoantigen may be related to IMN. Sethi et al. [22] reported neural epidermal growth factor-like 1 protein (NELL-1), a protein detected in the glomeruli of PLA2R-negative MN patients. In this study, 34 of 210 PLA2R-negative biopsies were NELL-1-positive (16\%). Uniform staining of NELL-1 along the GBM and its subepithelial deposition suggested that this protein might be derived from podocytes rather than circulating entrapped antigens or immune complexes. There was no staining of NELL-1 in the mesangium or subendothelial areas, which indicated that NELL-1 was unlikely to fall off from the mesangial cells or endothelial cells. It is unlikely that NELL-1 is shed from mesangial cells or endothelial cells because there was no mesangial or subendothelial staining in the NELL-1-positive MN. NELL-1-related MN may be a distinct type of primary $\mathrm{MN}$.

\section{Planting Antigen}

In 1982, Border et al. [23] injected New Zealand white rabbits intravenously with cationic bovine serum albumin (BSA), resulting in the formation of subepithelial immune complexes in the glomerulus, thereby establishing an experimental animal model of $\mathrm{MN}$ induced by cationic BSA. In contrast, the injection of both anionic and native BSA resulted in mesangial deposits instead of subepithelial deposits. It indicates that antigenic charge can play an important role in $\mathrm{MN}$ immune complex formation. In daily life, BSA comes from milk or beef protein. After ingesting these foods, some undigested proteins can cross the intestinal barrier and enter the blood, inducing the formation of serum anti-BSA antibodies. Therefore, BSA may become the exogenous food antigen of $\mathrm{MN}$, especially in children. In 2011, Debiec et al. [24] tested glo- merular circulating anti-BSA antibodies, and BSA in serum and in glomerular immune complex depositions among 50 patients with $\mathrm{MN}$ and 172 healthy controls by ELISA and the WB method. They found four children and 7 adult patients with MN had high serum BSA levels and high circulating anti-BSA IgG1/IgG4 antibody levels, while BSA in the glomerular immune complex deposition was found four children, which co-locates with specific anti-BSA IgG antibody at the glomerular epithelial space. The study also found that serum BSA in four children was cationic, while it was neutral in seven adults. It suggests such cationic BSA could be a target antigen of child MN. This antigen combines with negatively charged glomerular capillary wall and planting antigens, forms immune complexes with circulating anti-BSA antibodies, and finally leads to the occurrence of MN (Fig. 1b).

\section{Complement System Activation}

Studies have revealed that IgG4 is the main subtype of IMN antibodies $[4,12,20]$. Because IgG4 cannot activate the classical complement pathway, $\mathrm{Clq}$ and other molecules of the classical complement pathway are rare in IMN patients. At the same time, due to the lack of galactosidase, IgG4 can bind to the molecular mannose-binding lectin (MBL) of the MBL pathway, so this pathway is considered as the main complement activation pathway in IMN patients [25]. However, IMN can also occur in patients with deficiency of the MBL pathway caused by some genetic defects, suggesting that the complement bypass pathway may also be involved in the pathogenesis of IMN [26]. But Chi et al. [27] found C1q in the glomerulus of many patients who were anti-PLA2R antibodies positive. $\mathrm{Clq}$ is a molecule in the classical complement pathway, suggesting that the pathogenesis of IMN may include other complement system activation pathways besides IgG4-mediated pathways. They also found the plasma C5a concentration was predictive of the 12-month remission in $\mathrm{MN}$ patients. C5a is a potent inflammatory character; it can provide both co-stimulatory and survival signals to naive CD4+ T cells and enhance effector $\mathrm{T}$-cell expansion. It also regulates dendritic cell function. Lower circulatory C5a may be associated with less stimulation of inflammatory cells. In addition, the C5b-9 membrane attack complex forms after the activation of complement. It induces the production of reactive oxygen species, leading to local lipid peroxidation, GBM damage, and proteinuria. On the other hand, it induces apoptosis and exfoliation of podocytes, leading to reduced number of glomerular podocytes [28] (Fig. 1c). 


\section{Research Progress in Genetic Pathogenesis}

In recent years, with the development of genomics, research on the genetic pathogenesis of IMN has been further developed. Up to now, multiple susceptibility genes and their single nucleotide polymorphisms (SNPs) have been found to be associated with IMN. SNP refers to the DNA sequence polymorphism caused by single nucleotide variation on the genome level. SNP is closely related to human susceptibility to diseases, phenotypic differences, and drug sensitivity.

\section{PLA2R1 Gene Polymorphism}

The PLA2R1 gene is located on the q23-q24 region of human chromosome 2. Its product is expressed in glomerular podocytes. PLA2R is a type I transmembrane receptor which belongs to the mannose receptor family.

In a European population, Stanescu et al. [29] pioneered the use of a genome-wide association study (GWAS) to analyze the whole-genome sequencing of IMN patients and healthy controls in three different centers from Britain, France, and the Netherlands. After population stratification analysis and the center joint analysis, they found that the SNP loci of PLA2R1 gene intron 1 rs4664308 was significantly associated with IMN ( $p=$ $\left.8.6 \times 10^{-29}\right)$. Bullich et al. [30] verified this finding in a Spanish population in 2014. By sequencing the PLA2R1 gene of 89 IMN patients and 286 healthy controls, they found that rs4664308 had a strong correlation with IMN $(p=0.005)$ and that the gene polymorphism could predict the response of IMN patients to immunosuppressive therapy and disease prognosis. In American Caucasians [31], the PLA2R1 SNP rs35771982 was most strongly associated with PLA2R-positive MN, and its association was further increased in the joint analysis: rs3749117 was in full linkage disequilibrium with rs35771982 and had the next best association with MN. PLA2R1 SNPs were not found to be associated in American Africans with either PLA2R-positive MN or PLA2R-negative MN. Possibly, the low frequency of the G-allele (7\%) in this American African population combined with the smaller sample size did not provide adequate power to detect association.

In an Asian population, Kim et al. [32] genotyped 199 patients with IMN, 33 patients with SMN, and 356 subjects with normal blood pressure and no proteinuria in South Korea. The allele frequencies of C in rs35771982 and $G$ in rs3828323 were 73.6 and $73.9 \%$ in all subjects, respectively. Subjects with the CC genotype in rs35771982 had a higher susceptibility to IMN than subjects with other genotypes. Patients with SMN were not different from controls regarding the PLA2R genotype. No impact of genetic polymorphisms on renal survival was detected. Ramachandran et al. [33] studied 114 adult IMN patients and 95 healthy controls in India. Anti-PLA2R antibody was estimated before treatment and after 6 and 12 months of therapy. Enhanced glomerular staining for PLA2R was assessed on fresh frozen tissue. Genotype analysis was done on them using TaqMan assays for six SNPs. The results showed that the SNPs rs3749119, rs3749117, and rs4664308 in PLA2R1 were significantly associated with IMN. Patients with a high-risk genotype had higher antiPLA2R levels. In Japan, Thiri et al. [34] genotyped across the $P L A 2 R 1$ gene, using $183 \mathrm{IMN}$ patients and 811 healthy controls, and found five SNPs around the PLA2R1 gene were significantly associated with IMN. These are three SNPs previously reported to be associated with IMN, rs3749119, rs35771982, and rs 1511223, and two novel intronic SNPs (rs2715928 and rs16844715). In the study by Kaga et al. [35], among 6 SNPs within PLA2R1, 3 SNPs (rs3749117, rs35771982, and rs2715918) were significantly associated with IMN in their Japanese cohort.

In China, Lv et al. [36] selected 1,112 IMN patients and 1,020 healthy controls to study the rs4664308 locus of the PLA2R1 gene and the other two rs35771982 and rs3749117 loci located in exon 5. These three SNPs were found to be significantly correlated with IMN in their study. Guangyu et al. [37] also found that the frequency of rs35771982 is significantly higher than healthy controls among China's northeast Han people with IMN. They speculated that the locus mutation resulted in histidine being replaced with aspartic acid, which then led to changes in the CTLD structure of PLA2R molecules. These changes exposed the antigen epitope and stimulated the body to produce PLA2R antibodies, causing immune damage. Wang et al. [38] found that in IMN patients with risk genotype combinations of SNP loci such as rs2715918 (GA/AA), rs4665143 (GA/AA) on the PLA2R gene, and rs2187668 (GA/AA) on the HLA-DOA1 gene, the risk of MN progression increased 10.61 times. These three are likely to play a combined role in the progression of IMN patients through their respective biological pathways.

\section{HLA Gene Polymorphism}

The HLA gene is located on the p21.31 region of human chromosome 6 , and 239 gene loci have been found. Traditionally, HLA genes are classified into class I, II, and III genes according to their loci and characteristics. HLA$B, H L A-C$, and HLA-A are classical HLA-I genes, while $H L A-D P, H L A-D Q$, and HLA-DR are classical HLA-II genes. They are all highly polymorphic. 
Stanescu et al. [29] found that the SNP loci rs2187668 of HLA-DQA1 gene intron 1 was also significantly correlated with IMN in a Spanish population. The interaction between rs 2187668 and rs4664308 mentioned above jointly determined the genetic susceptibility of IMN. The incidence of IMN increased 77.46 times in the population carrying the risk genotype combination of these two SNP loci (rs2187668[AA] + rs4664308[AA]) compared with that in the population carrying the protective genotype combination (rs2187668[GG] + rs4664308[GG]). The study by Saeed et al. [31] confirmed the previously observed stronger association with $H L A-D Q A 1$ than with PLA2R1 variants. Interestingly, the HLA-DQA1 SNP rs2187668 was strongly associated with PLA2R-positive $\mathrm{MN}$ in American Caucasians. However, the strength of its association substantially decreased in PLA2R-negative MN. In American Africans, rs2187668 was associated with PLA2R-positive MN but not with PLA2R-negative MN. In addition, in a study of Chinese population, Lv et al. [36] also discovered that SNP rs2187668 in HLADQA1 showed a strong association with IMN. In patients with both HLA-DQA1 and PLA2R1 risk genotypes, antiPLA2R antibodies were present in the circulation in $73 \%$ of patients and in the glomerulus in $75 \%$ of patients. However, in patients with both HLA-DQA1 and PLA2R1 protective genotypes, circulating and glomerular antibodies barely existed. In India, Ramachandran et al. [33] have also found SNP rs2187668 within HLA-DQA1 was significantly associated with IMN; it was also associated with anti-PLA2R positivity in an Indian cohort. In a Japanese population, Kaga et al. [35] made the same discovery that intronic SNP rs2187668 within HLA-DQA1 was significantly associated with IMN in their cohort even after the Bonferroni correction. Actually, the results have already been demonstrated in European, Chinese, Caucasian, and African American cohorts positive for antiPLA2R antibodies, and an Indian cohort.

Liu's team [39] have found that PLA2R-related MN patients had two $H L A$ risk alleles $H L A-D R B 1{ }^{*} 15: 01$ and $H L A-D R B 3{ }^{*} 02: 02$ by sequencing analysis of the whole major histocompatibility complex (MHC) region. HLA$D R B 1 * 15: 01$ was found in $81.8 \%$ of PLA2R-related MN patients and $21 \%$ of healthy controls. HLA-DRB3 ${ }^{*} 02: 02$ was present in $60.6 \%$ of PLA2R-related MN patients and $28 \%$ of healthy controls. The two HLA risk alleles are strongly associated with increased risk of PLA2R-related MN. After correcting for those two alleles, no other alleles were significantly associated with PLA2R-related MN. Wang et al. [40] found that $H L A-D R B 1 * 1502$ was not a risk allele of IMN but was related to the anti-PLA2R antibody level and disease severity. It was the only allele related to renal prognosis, and patients with this allele had a higher rate of progression to end-stage kidney disease and lower renal survival rate. The interaction between HLA-DRB1 * 1502 and HLA-DRB ${ }^{*} 0301$ increases the risk of patients with high titers of anti-PLA2R antibody by 5.5 times. HLA-DRB1 ${ }^{*} 0301$ is closely related to higher levels of anti-PLA2R antibodies, suggesting that the encoded MHCII molecule played an important role in the presentation of PLA2R antigen to the immune system.

\section{Myosin Heavy Chain 9 Gene Polymorphism}

The myosin heavy chain 9 (MYH9) gene is located on the $\mathrm{q} 12$ region of human chromosome 22 , composed of 41 exons, its $\mathrm{N}$ initial domain, and the $\mathrm{C}$-terminal domain coding nonmuscle myosin IIA (NMMHC-IIA) heavy chains, which is expressed in glomerular podocytes and mesangial cells. In a Taiwan population, Chen et al. [41] first reported that $M Y H 9$ gene polymorphism was closely related to IMN. The results of MYH9 gene sequencing among 135 IMN patients and 265 healthy controls showed that AA genotype frequency at rs12107 in the $3^{\prime}$ noncoding region was significantly increased in the IMN group, and the difference was statistically significant $(p=0.021)$. The C-A haplotype was also the susceptible haplotype of IMN in the Taiwan population $(p=0.04)$.

\section{Signal Transducer and Activator of Transcription 4 Gene Polymorphism}

The signal transducer and activator of transcription 4 (STAT4) gene, located on the q32.2-32.3 region of human chromosome 2 , contains a total of 24 exons. It can encode transcription factors and plays an important role in the inflammatory response of various immune-mediated diseases. At the same time, STAT4 is involved in the regulation of the immune response by transmitting transcriptional signals activated by IL-12, IL-23, IFN1, and other cytokines. In recent years, studies have found that rs75748665 of the STAT4 gene in intron 3 is closely related to rheumatoid arthritis, SLE, sclerosis, type 1 diabetes, systemic sclerosis, and other autoimmune diseases [4245]. In order to explore the correlation between STAT4 and IMN, Chen et al. [46] selected 138 patients with IMN and 265 healthy controls from the Chinese Taiwan population and detected three SNP sites of STAT4, rs3024908, rs3024912, and rs3024877. They found that IMN patients with GG genotype at rs3024908 were more likely to develop renal failure. At the same time, Chen et al. [47] also found that IL-6, TLR-4, and TLR-9, which are related to 
inflammatory response, are also related to the susceptibility of IMN. Multiple SNP loci have been confirmed in Taiwan population.

\section{Tumor Necrosis Factor- $\alpha$ Gene Polymorphism}

The tumor necrosis factor- $\alpha(T N F-\alpha)$ coding gene located on the 21.3 region of human chromosome 6 , namely, the $M H C$ genes of the HLA-III area, contains four exons. Early animal experiments have shown that TNF- $\alpha$ can induce glomerular injury. In vivo studies have also found that plasma and urine samples of patients with $\mathrm{MN}$ have correspondingly increased TNF- $\alpha$ levels [48]. Neale et al. [49] found that TNF- $\alpha$ was increased in both the glomerular epithelial cells and the GBM in MN patients, suggesting that TNF- $\alpha$ is associated with the pathogenesis of MN. In 2006, Bantis et al. [50] found that TNF- $\alpha$ gene G-308A polymorphism was a susceptibility factor for IMN, and the mutation of guanine to adenine at this locus produced a new allele TNFA2. In 2007, Thibaudin et al. [51] studied 100 cases of IMN patients and 232 healthy controls and found that TNF- $\alpha$ gene G-308A polymorphism and TNFd gene polymorphism led to the increase of TNF and promoted the incidence of IMN. TNFA2 and $T N F d 2$ were the risk alleles of the disease, respectively.

\section{NPHS1 Gene Polymorphism}

The NPHS1 gene is located on the q13.1 region of human chromosome 19, encoding nephrin, a protein specifically expressed in the glomerular fissure membrane. Nephrin belongs to the Ig family and is a transmembrane protein. The interaction of nephrin molecules between adjacent podocytes forms a "pull-chain structure" at the slit diaphragm, which plays an important role in maintaining the integrity of the glomerular filtration barrier and regulating renal function. Zhanyun et al. [52] found that the expression of nephrin in IMN was weakened, suggesting that immune-mediated podocyte injury may lead to the decrease of nephrin in podocytes, and thus participating in the occurrence of IMN proteinuria. Lo et al. [53] studied a Taiwan population and found that rs437168 located in exon 17 of the NPHS1 gene was significantly different between the IMN group and the healthy control group $(p=0.031)$, and the frequency of $\mathrm{G}$ allele was significantly increased in IMN patients. Stratified analysis showed that the GG genotype was associated with a low remission rate.

\section{Other Gene Polymorphisms}

Studies have shown that there is a correlation between the $4 \mathrm{G}$ allele at the 675 locus in the initiator region of the
Table 1. Gene polymorphisms associated with IMN

\begin{tabular}{ll}
\hline $\begin{array}{l}\text { Gene } \\
\text { polymorphism }\end{array}$ & SNP \\
\hline PLA2R1 & $\begin{array}{l}\text { rs4664308, rs35771982, rs3749117, rs3749119, } \\
\text { rs4664308, rs1511223, rs16844715, rs2715928, } \\
\text { rs2715918, rs4665143 }\end{array}$ \\
\hline HLA-DQ1 & rs2187668 \\
\hline MYH9 & rs12107 \\
\hline STAT4 & rs3024908 \\
\hline NPHS1 & rs437168 \\
\hline
\end{tabular}

SNP, single nucleotide polymorphism; IMN, idiopathic membranous nephropathy; PLA2R1, phospholipase A2 receptor 1; HLA, human leukocyte antigen; STAT4, signal transducer and activator of transcription 4; MYH9, myosin heavy chain 9.

plasminogen activator inhibitor-1 (PAI-1) gene and some events, including renal function deterioration and increased cardiovascular events in IMN patients [54]. Patients with the CC genotype in the $u P A$ gene $3^{\prime}$ noncoding region have an increased risk of ESRD and tumor [55]. This indicated that the polymorphism of PAI-1 and $u P A$ genes was closely related to the clinical manifestations and prognosis of IMN. In brief, with the wide application of GWAS and high-throughput sequencing technology, people are more and more aware of the genetic pathogenesis of IMN. Table 1 summarizes the gene polymorphisms currently found to be related to the pathogenesis of IMN.

\section{Application of PLA2R and Its Antibodies in the Diagnosis and Treatment of IMN}

\section{The Diagnostic Value of PLA2R and Its Antibody in IMN}

Beck et al. [4] first discovered the role of PLA2R in the pathogenesis of IMN in 2009, and subsequently a series of clinical studies verified the sensitivity and specificity of serum anti-PLA2R antibodies. In May 2014, FDA approved the anti-PLA2R antibody test kit for clinical use, thus opening a new chapter in IMN diagnosis. Currently, immunofluorescence assay (IFA) and ELISA are widely used, which can achieve the qualitative and quantitative detection of serum antibodies. There are three methods to detect anti-PLA2R antibody [56], including WB, IFA, and ELISA. When using ELISA, a cutoff value is used to define PLA2R antibody positivity. Different studies used 
different cutoff values, $14 \mathrm{RU} / \mathrm{mL}$ or $20 \mathrm{RU} / \mathrm{mL}$. Even with a lower cutoff value, ELISA is not as sensitive as IFA. Bobart et al. [57] conducted a retrospective study, in which the patients were screened by laboratory and imaging examinations to eliminate the underlying causes of SMN. They found that patients with ELISA titer $>20 \mathrm{RU} /$ $\mathrm{mL}$, eGFR $>60 \mathrm{~mL} / \mathrm{min}$, or $2 \mathrm{RU} / \mathrm{mL} \leq$ ELISA titer $\leq 20$ $\mathrm{RU} / \mathrm{mL}$, and positive IFA were confirmed by renal biopsy for MN. In addition, all patients with positive IFA were MN regardless of ELISA titer. Therefore, IFA is more sensitive than ELISA. The PLA2R-related IMN could be diagnosed according the result of ELISA titer or IFA, and the time of renal biopsy could be delayed.

As we all know, renal biopsy is a traumatic examination, has some contraindications, and also requires the hospital to have certain puncture conditions. Can the detection of anti-PLA2R antibody replace renal biopsy to help us diagnose? Bobart et al. [57] found that of 132 patients with positive anti-PLA2R antibodies, 129 were diagnosed with $\mathrm{MN}$ in subsequent renal biopsies. A meta-analysis of 15 studies showed that the sensitivity of anti-PLA2R antibody detection was about $78 \%$ and the specificity was up to $99 \%$ [58]. Such high specificity not only ensures the accuracy of the diagnosis of IMN but also provides a diagnostic reference for those patients who cannot accept renal biopsy with poor physical conditions and many complications. The overall positive rate of anti-PLA2R antibody in IMN patients in early clinical studies was between 70 and $80 \%$, and some recent studies from different ethnic cohorts have shown a lower positive rate. Kaga et al. [59] reported that among 217 IMN patients in Japan, only 73 cases were positive for anti-PLA2R antibody, with a positive rate of $33.6 \%$. This may be related to the fact that the subjects recruited in the earlier study had mostly severe proteinuria, while the patients in the recent study cohort had mostly mild proteinuria. Anti-PLA2R antibody may also be undetectable in the remission phase or early stage of the disease $[60$, 61]. Studies have shown that serum anti-PLA2R antibody is indeed absent at the time of onset and can be measured during follow-up. This may be due to the "kidney as sink" phenomenon. The anti-PLA2R antibodies bind to the antigen in the kidney with high affinity and can only be detectable when the binding sites in the kidney are saturated [56]. It reminds us that although antiPLA2R antibody is an excellent diagnosis index for IMN, it still has its limitation. Anyway, it might provide severe IMN patients with an explicit diagnosis without renal biopsy, protecting these patients from biopsy complications. It is also an effective method to help diagnose $\mathrm{MN}$ in some basic hospitals that are not yet qualified to carry out renal biopsy.

In recent years, PLA2R antigen detection has also been carried out in renal biopsy tissues. Since paraffin-embedded renal biopsy specimens and fresh frozen sections can be used for detection, both retrospective study and realtime diagnosis of IMN can be achieved. Renal biopsy tissue PLA2R antigen-positive result was found in most IMN patients, but PLA2R antigen-positive results can also be seen in a few patients with SMN, especially in tumors, lupus nephritis, sarcoidosis, hepatitis B-associated $\mathrm{MN}$, etc. [62]. PLA2R antigen-negative result was seen in a few IMN patients (possibly there are other antigens such as THSD7A) or in most SMN patients. Combined with the literature reports, the sensitivity of PLA2R antigen detection for renal biopsy is between 70 and 92\% [60]. Circulating anti-PLA2R antibodies may be negative in some situation, such as when the time from the onset of $\mathrm{MN}$ to renal biopsy is too long, during disease immune remission, when the affinity between antigen and antibody is strong, or when some other reasons lead to fast antibody clearance. However, in these situations, the PLA2R antigen can still be detected in the kidney, indicating that it has a higher sensitivity $[61,63]$. Therefore, combined detection of PLA2R antigen and antibody is expected to improve the diagnostic efficiency of IMN.

\section{The Relationship between Anti-PLA2R Antibody and IMN Activity}

Many studies in recent years have shown that serum anti-PLA2R antibodies were closely related to IMN disease activity. Hofstra et al. [64] followed up 12 IMN patients for up to 45 months and found that the serum antibody titer was higher in the early stage of the disease with massive proteinuria, and it also decreased significantly or disappeared completely in remission of the disease and gradually increased in the recurrence of the disease. The serum antibody titer was significantly correlated with the proteinuria level after correction of the $\operatorname{IgG}$ excretion fraction. Beck et al. [65] followed up 35 IMN patients with MabThera treatment for 2 years and found changes in the serum anti-PLA2R antibody levels in IMN patients often prior to changes in urine protein levels, suggesting immunologic remission was ahead of clinical remission and immunologic recurrence was ahead of the clinical recurrence. Therefore, the serum antibody levels were more accurate in time to reflect the activity of the disease than the proteinuria levels. Clearance of the glomerular immune complex and reconstruction of the glomerular structure, or its deposition and destruction, both 
take some time. Therefore, the change of the proteinuria level is later than the change of the antibody level. $\mathrm{Wu}$ et al. [66] also found that the antibody turned negative in IMN patients with complete remission and decreased significantly in patients with partial remission, suggesting that the change of the anti-PLA2R antibody level in IMN patients is closely related to the status of IMN: if the antibody level decreased, the condition was improved; if the serum antibody level continues to be positive or quantitatively elevated, the disease is not in remission or has relapsed.

\section{The Role of Anti-PLA2R Antibody in MN Treatment}

On one hand, whether the anti-PLA2R antibody is positive or not may directly influence the implementation of immunosuppressive therapy. A meta-analysis involving 190 patients by $\mathrm{Wu}$ et al. [67] found that the spontaneous remission rate of patients with serum anti-PLA2R antibody negative was significantly higher than that of patients with serum antibody positive. Therefore, in order to avoid the malignant events caused by immunosuppressive therapy, it may be more beneficial to adopt conservative treatment for patients with initial negative serum anti-PLA2R antibody. The review by Rodas et al. [68] also mentioned that MN patients with anti-PLA2R antibody level $<40 \mathrm{IU} / \mathrm{mL}$ can benefit from conservative treatment, so as to avoid the side effects of immunosuppressive therapy. This titer standard can be used as a tool for selection of treatment.

On the other hand, since the serum antibody level can reflect the activity of the disease earlier than proteinuria level, it is of great significance to combine the analysis of these two to guide the treatment. Traditional MN treatment regimens include ACEI/ARB and other conservative treatments, alkylating agents (nitrogen mustard and cyclophosphamide) combined with glucocorticoid, calcineurin inhibitors (cyclosporine and tacrolimus), and other immunosuppressive treatments. Cattran et al. [69] gave treatment suggestions for different patients on whether to use conservative treatment and when to start immunosuppressive treatment: patients with mild proteinuria ( $<4 \mathrm{~g} /$ day), low serum anti-PLA2R antibody lev$\mathrm{el}$, and normal renal function can only receive conservative treatment such as $\mathrm{ACEI} / \mathrm{ARB}$ and regular monitoring of the urinary protein level; patients with moderate proteinuria ( $4 \mathrm{~g} /$ day $<$ and $<8 \mathrm{~g}$ /day), moderate serum antiPLA2R antibody level, and normal renal function can be observed with conservative treatment for 6 months. Patients with massive proteinuria ( $8 \mathrm{~g} /$ day), high serum anti-PLA2R antibody level, and with or without renal func- tion decline were recommended to be treated conservatively for 3 months. If patients' proteinuria is $\geq 4 \mathrm{~g} /$ day, persistent, cytotoxic + steroids or calcineurin inhibitor or rituximab should be given. When immunosuppressive treatment is given and it did not work, consider replacing with another immunosuppressant. De Vriese et al. [70] proposed an individualized IMN treatment based on serum antibody levels: (1) for patients with high baseline antibody levels, it was recommended to monitor serum anti-PLA2R antibody levels monthly and start immunosuppressive therapy if there is no significant decrease or continuous increase. (2) For patients with high baseline antibody level which decreased after treatment or patients with low or moderate baseline antibody level, it was recommended to monitor serum antibody levels and proteinuria level every 2 months. If the subsequent antibody level no longer continues to decline, it was recommended to start immunosuppressive therapy; if the antibody level decreased gradually and successively, it was recommended to continue to monitor serum antibody levels and albuminuria levels every 2 months, until antibody turns negative or albuminuria remission. Therefore, whether anti-PLA2R antibody levels were decreased or whether proteinuria was reduced after treatment can help to determine patients' response to treatment, and has guiding significance for adjusting drug dose or treatment regimen.

For patients with $\mathrm{MN}$, glucocorticoid combined with alkylation is more effective than conservative treatment or glucocorticoid alone, but it increases the risk of bone marrow suppression, infection, and cancer. Calcineurin inhibitors improved proteinuria but increased renal toxicity. Many patients relapse after discontinuation of medicine and develop drug dependence. Long-term use of immunosuppressive agents also increases renal toxicity. Therefore, traditional immunosuppressive agents have many side effects when treating the primary disease. Research on podocyte autoantigens and antibodies has promoted the development of new targeted drugs, and it is expected to bring good news to MN patients. The use of specific monoclonal antibodies to block the production of autoantibodies and the deposition of subepithelial immune complexes will be a new therapeutic target. The review published in Nature Review Nephrology by Ruggenenti et al. [71] pointed out that B-cell regulatory therapy or plasma cell-targeted therapy, represented by rituximab, can block pre-B-cell migration to activated B cells and lead to apoptosis of plasma cells that produce antiPLA2R or THSD7A antibodies, effectively reducing the level of B cells and these two circulating antibodies, so as 
to alleviate $\mathrm{MN}$. In addition, belimumab, a monoclonal antibody, prevented B cells from differentiating into plasma cells, and bortezomib, a protease inhibitor, had a similar effect by inducing apoptosis of plasma cells. However, large sample clinical trial data of these new drugs were still lacking, and their effectiveness and safety still needed to be further verified by follow-up studies.

\section{The Prognostic Value of Anti-PLA2R Antibody in IMN}

Hoxha et al. [72] observed IMN patients receiving immunosuppressive therapy for up to 2 years and found that patients with higher baseline antibody levels had a higher rate of persistent nonremission of proteinuria. They required longer time to achieve complete remission and had a higher risk of renal function deterioration progression. For the antibody level at the end of the follow-up, proteinuria was the lowest in the complete remission group, followed by the partial remission group, and the highest in the continuous nonremission group. Multivariate Cox regression analysis showed that the anti-PLA2R antibody level was an independent risk factor for persistent nonremission of proteinuria. Pourcine et al. [73] conducted a follow-up of $108 \mathrm{MN}$ patients for up to 14 years and found that patients with higher baseline antibody and proteinuria levels in PLA2R-associated MN had a higher risk of progressing to stage $4 \mathrm{CKD}$ and beyond. Recently, studies by De Vriese et al. [70] also showed that dynamic monitoring of serum antibody levels can reliably predict patients' response to treatment and long-term prognosis of the disease: patients with low baseline antiPLA2R antibody levels or continuously decreased antibody levels after treatment have a high rate of spontaneous remission; patients with high baseline anti-PLA2R antibody levels or persistently elevated antibody levels after treatment often present with high levels of proteinuria and hypoalbuminemia, and are also more prone to renal dysfunction. The antibody level of patients with remission increased again, indicating the recurrence of the disease. The persistence or recurrence of serum antibodies after kidney transplantation suggested a recurrence of the primary disease.

In the clinical study by Qu et al. [74], a total of 359 INM patients were recruited, of which 248 were anti-PLA2R antibody positive. According to the antibody titer, they were divided into three groups by ELISA: low titer 20-63 $\mathrm{U} / \mathrm{mL}$, medium titer $64-179 \mathrm{U} / \mathrm{mL}$, and high titer $179 \mathrm{U} /$ $\mathrm{mL}$. In the moderate and high titer groups, the risk of nonremission after treatment was increased, and they were more difficult to achieve spontaneous remission. In the high titer group, the risk of progression to renal insufficiency was significantly increased. The recurrence rate was also higher in patients who remained positive for the antibody. Although the study samples were all from the Chinese population receiving immunosuppressive agents, risk stratification of the prognosis was like that of the previous European population receiving rituximab, suggesting that this stratification of prognosis also applies to the Chinese population. Song et al. [75] also found that in anti-PLA2R antibody-negative patients, the spontaneous remission rate was significantly higher than that in antibody-positive patients. Their time for achieving remission was shorter than that in the latter. The antiPLA2R antibody positivity caused patients to be at higher risk for progress to stage $3 \mathrm{CKD}$ or beyond; it is an independent risk factor for progression to CKD. Above all, it is of great value to take advantage of anti-PLA2R antibody for MN prognosis evaluation.

\section{Thrombotic Events in MN and Its Management}

Already in the early 1980s it was noted that the risk of a venous thrombotic event (VTE) and associated pulmonary embolism was particularly high in patients with nephrotic syndrome (NS) due to primary MN. VTEs including deep venous thrombosis, renal vein thrombosis, and pulmonary embolism are recognized as early complications of primary $\mathrm{MN}$ that carry significant morbidity and mortality [76, 77]. A European study [78] has found that clinically apparent venous thromboembolic events occur in about $7 \%$ of patients with MN. Hypoalbuminemia, particularly $<2.8 \mathrm{~g} / \mathrm{dL}$, is the most significant independent predictor of venous thrombotic risk. It was reported in a Chinese study [79] that $36 \%$ of primary $\mathrm{MN}$ patients had a VTE, 33\% had renal vein thrombosis, and $17 \%$ had a pulmonary embolism. The morbidity of VTEs from different studies ranged from 3 to $48 \%$ in patients with NS [80].

Next to VTE, patients with NS might also be at risk of arterial thrombotic events (ATEs). The primary cardiovascular events (CVEs) included acute coronary syndrome and ischemic stroke $[76,77]$. In the study by Lee et al., the morbidity of CVEs was 4.4, 5.4, 8.2, and $8.8 \%$ at $1,2,3$, and 5 years, respectively, in a primary MN cohort [81]. In addition, they found that the incidence of CVEs was at least as high as that of ESRD early in the course of the disease. Therefore, a reduction in CVEs should be considered as a focus of intervention and as a therapeutic outcome measure in primary MN. In the study by Zou et 
al. [80], the cumulative incidence of ATEs was relative lower than that in Lee's study, and also ischemic stroke accounted for $45 \%$ of the ATEs, suggesting the Chinese cohort was distinguished from the western countries, where the majority of ATEs was CHD.

Hofstra et al. [76] suggested that it is evident that prophylactic warfarin should be used in patients with serum albumin $<2.5 \mathrm{~g} / \mathrm{dL}$ and low bleeding risk. It is important to realize that $43 \%$ of patients with a VTE in the study of Lionaki et al. [78] were on aspirin when the VTE developed. However, in patients with high bleeding risk and in patients with NS, but low calculated VTE risk, aspirin therapy might be offered. In view of the potential prothrombotic effects of corticosteroids, it is important that the decision to start immunosuppressive therapy (often including high dose of prednisone) should not lead to postponement of the start of aspirin. Recently, Zou et al. [77] identified 65 primary studies addressing prophylactic and therapeutic anticoagulation therapy in primary $\mathrm{MN}$ and found that personalized prophylactic aspirin or warfarin could be considered to prevent ATEs and VTEs in primary $\mathrm{MN}$ patients with serum albumin $<3.2 \mathrm{~g} / \mathrm{dL}$. Moreover, the treatment regimen of thromboembolic complications in primary $\mathrm{MN}$ patients was similar to that in the general population with thromboembolic events. Since more evidence indicated that DOACs provided an effective and safe regimen for anticoagulant treatment in NS compared to conventional therapy, they might be promising anticoagulants in primary MN. Finally, patients should continue the previous primary $\mathrm{MN}$ treatment protocol during the entire treatment period until they achieve remission, the protocol is completed, and the underlying diseases have resolved.

\section{The Novel Finding of SMN}

Although anti-PLA2R antibodies are present in the serum of most patients with IMN, a few patients with SMN also have positive results of serum anti-PLA2R antibodies. In the study of Radice et al. [82], a total of 252 consecutive IMN patients, 184 pathological, and 43 healthy controls were tested for anti-PLA2R antibody using indirect immunofluorescence. Anti-PLA2R autoantibodies were detectable in $178 / 252$ of IMN patients, $1 / 80$ of primary GN, 0/72 of secondary GN, 9/32 of SMN, and 0/43 of healthy controls. Only 10 disease control patients resulted positive. Of note, 9 of them had $\mathrm{MN}$-associated disease which was known to cause (or be associated with) a secondary form of MN (mainly malignancies). This finding is especially important for patients with $\mathrm{MN}$ associated with malignancies, but causal relationship between tumors and anti-PLA2R-induced MN remains to be established, and whether these cases represent true SMN or rather PLA2R-associated MN with coincident secondary disease is not known. Garcia-Vives et al. [83] tested anti-PLA2R antibodies in 109 SLE patients, including 37 patients with lupus MN. They found that there are 10 cases anti-PLA2R antibody positive, including 7 cases with lupus $\mathrm{MN}$, suggesting there was low popularity of PLA2R in MN complicated by SLE, but the specific IgG anti-PLA2R antibody was absent in these patients.

A new antigen associated with SMN was also found. Sethi et al. [84] identified two novel proteins, exostosin-1 (EXT1) and exostosin-2 (EXT2), that accumulate along the GBM in a subset of PLA2R-negative MN. The staining pattern mirrors the granular IgG staining along the GBM, indicating that EXT1 and EXT2 are the likely antigens in the immune complexes. Most importantly, the clinical features and kidney biopsy findings show that EXT1/ EXT2-associated MN is mostly present in patients with autoimmune manifestations or diseases including lupus, but absent in all PLA2R-associated MN and control cases. Taken together, these findings show that EXT1 and EXT2 represent novel biomarker proteins and possibly the target antigens in SMN. Further, Tian et al. [85] have found that 3 SNPs within PLA2R1 (rs35771982, rs3749117, and rs4664308) were associated with increased susceptibility to IMN and that there was also a strong association of rs4664308 with susceptibility to SMN. These findings may help identify individuals with increased risk for IMN and SMN so that clinicians can perform early interventions and make better treatment decisions. Above all, anti-PLA2R antibody has a low popularity in SMN patients; the new antigens EXT1 and EXT2 involved with SMN, and novel SNPs associated with SMN were gradually found. The finding might provide people novel ways to diagnose and treat SMN, and help us to know this disease better, to distinguish SMN from IMN, and to make better and different therapies for them. Although these recent advances are changing people's understanding of IMN and SMN, further studies are still needed to explore the relationship between IMN and SMN.

\section{Conclusion and Prospect}

All in all, people have found that podocyte autoantigens, planting antigen, complement activation on the molecular level, and gene polymorphism on the genetic 
Table 2. Pathogenesis of IMN

\begin{tabular}{ll}
\hline Podocytes' autoantigens & PLA2R, THSD7A, NEP, SOD2, AR, NELL-1 \\
\hline Planting antigens & Cationic BSA \\
Complement system activation & Classical pathway, MBL pathway, alternative pathway \\
Gene polymorphism & PLA2R1, HLA, MYH9, STAT4, TNF- $\alpha$, NPHS1, PAL- 1, uPA \\
\hline
\end{tabular}

IMN, idiopathic membranous nephropathy; THSD7A, thrombospondin type-1 domain-containing 7A; NEP, neutral endopeptidase; SOD2, superoxide dismutase 2; AR, aldose reductase; NELL-1, neural epidermal growth factor-like 1 protein; BSA, bovine serum albumin; MBL, mannose-binding lectin; PLA2R1, phospholipase A2 receptor 1; HLA, human leukocyte antigen; MYH9, myosin heavy chain 9; STAT4, signal transducer and activator of transcription 4; TNF- $\alpha$, tumor necrosis factor- $\alpha$.

level consist of the pathogenesis of IMN in recent years (Table 2). Meanwhile, the low popularity of anti-PLA2R antibody in SMN patients, the new SMN antigens EXT1 and EXT2, and the novel SNPs associated with SMN were gradually found. These advances in the pathogenesis of IMN and SMN help us to know those two kinds of diseases and their differences better, increasing people's understanding of the kidney disease. The application of PLA2R and anti-PLA2R antibodies in IMN therapy is becoming the translational medicine model. However, the specific role of PLA2R, THSD7A, and other podocyte autoantigens and their antibodies in the pathogenesis of IMN remains to be further verified in subsequent animal models and podocyte models. IMN gene SNP researches explain the disease pathogenesis genetically, conforming to the trend of accurate medicine period. Follow-up researches based on race, multicenter, large sample, and prospective clinical study will help promote the clinical application of disease susceptibility genes. It is expected to offer new perspectives, for the gene diagnosis of disease, individualized treatment, and prognosis evaluation.

\section{Disclosure Statement}

The authors have no conflicts of interest to declare.

\section{Funding Sources}

This work was supported by grants from the National Natural Science Foundation of China (Nos. 81770736 and 30800532), the Doctoral Fund of the Ministry of Education of China (No. 200804871122), the Natural Science Foundation of Hubei Province (Nos. 2015CFB467 and 2014CFB197), the Chinese Medical Association (No. 14050510588), the Huazhong University of Science and Technology (Nos. 2015LC033 and 2015QN198), and the Research-based clinician scheme of Tongji Medical College, Huazhong University of Science and Technology.

\section{Author Contributions}

All the above authors have contributions to this review. The main contents of this review were analyzed and written by Zhifeng $\mathrm{Xu}$ and Lu Chen, assisted by Huiling Xiang. Chun Zhang and Jing Xiong provided guidance and revision.

\section{References}

1 Ronco P, Debiec H. Pathophysiological advances in membranous nephropathy: time for a shift in patient's care. Lancet. 2015;385 (9981):1983-92.

2 Chapter 7: Idiopathic membranous nephropathy. Kidney Int Suppl. 2012;2(2):186-97.

3 Xu X, Wang G, Chen N, Lu T, Nie S, Xu G, et al. Long-term exposure to air pollution and increased risk of membranous nephropathy inchina.J Am Soc Nephrol.2016;27(12):373946.

4 Beck LH Jr, Bonegio RG, Lambeau G, Beck DM, Powell DW, Cummins TD, et al. M-type phospholipase A2 receptor as target antigen in idiopathic membranous nephropathy. $\mathrm{N}$ Engl J Med. 2009;361(1):11-21.
5 von Haxthausen F, Reinhard L, Pinnschmidt HO, Rink M, Soave A, Hoxha E, et al. Antigen-specific IgG subclasses in primary and malignancy-associated membranous nephropathy. Front Immunol. 2018;9:3035.

6 Ancian P, Lambeau G, Mattéi MG, Lazdunski M. The human $180-\mathrm{kDa}$ receptor for secretory phospholipases A2. Molecular cloning, identification of a secreted soluble form, expression, and chromosomal localization. J Biol Chem. 1995;270(15):8963-70.
7 Kao L, Lam V, Waldman M, Glassock RJ, Zhu Q. Identification of the immunodominant epitope region in phospholipase A2 receptormediating autoantibody binding in idiopathic membranous nephropathy. J Am Soc Nephrol. 2015;26(2):291-301.

8 Fresquet M, Jowitt TA, Gummadova J, Collins R, O'Cualain R, McKenzie EA, et al. Identification of a major epitope recognized by PLA2R autoantibodies in primary membranous nephropathy. J Am Soc Nephrol. 2015;26(2):302-13.

9 Wang B, Zuo K, Wu Y, Huang Q, Qin WS Zeng $\mathrm{CH}$, et al. Correlation between B lymphocyte abnormality and disease activity in patients with idiopathic membranous nephropathy. J Int Med Res. 2011;39(1):86-95. 
10 Seitz-Polski B, Dolla G, Payré C, Girard CA, Polidori J, Zorzi K, et al. Epitope Spreading of autoantibody response to PLA2R associates with poor prognosis in membranous nephropathy. J Am Soc Nephrol. 2016;27(5):1517-33.

11 Seitz-Polski B, Debiec H, Rousseau A, Dahan K, Zaghrini C, Payré C, et al. Phospholipase A2 receptor 1 epitope spreading at baseline predicts reduced likelihood of remission of membranous nephropathy. J Am Soc Nephrol. 2018;29(2):401-8.

12 Tomas N, Beck L Jr, Meyer-Schwesinger C, Seitz-Polski B, Ma H, Zahner G, et al. Thrombospondin type-1 domain-containing 7A in idiopathic membranous nephropathy. $\mathrm{N}$ Engl J Med. 2014;371(24):2277-87.

13 Salant DJ. Unmet challenges in membranous nephropathy. Curr Opin Nephrol Hypertens. 2019;28(1):70-6.

14 Hoxha E, Wiech T, Stahl PR, Zahner G, Tomas NM, Meyer-Schwesinger C, et al. A Mechanism for cancer-associated membranous nephropathy. N Engl J Med. 2016; 374(20):1995-6.

15 Hara S, Tsuji T, Fukasawa Y, Hisano S, Morito $\mathrm{S}$, Hyodo T, et al. Clinicopathological characteristics of thrombospondin type 1 domaincontaining 7A-associated membranous nephropathy. Virchows Arch. 2019;474(6) 735-43.

16 Zaghrini C, Seitz-Polski B, Justino J, Dolla G, Payré C, Jourde-Chiche N, et al. Novel ELISA for thrombospondin type 1 domain-containing 7A autoantibodies in membranous nephropathy. Kidney Int. 2019;95(3):666-79.

17 Debiec H, Guigonis V, Mougenot B, Decobert F, Haymann JP, Bensman A, et al. Antenatal membranous glomerulonephritis due to anti-neutral endopeptidase antibodies. $\mathrm{N}$ Engl J Med. 2002;346(26):2053-60.

18 Debiec H, Nauta J, Coulet F, van der Burg M, Guigonis V, Schurmans T, et al. Role of truncating mutations in MME gene in fetomaternal alloimmunisation and antenatal glomerulopathies. Lancet. 2004;364(9441):1252-9.

19 Vivarelli M, Emma F, Pellé T, Gerken C, Pedicelli S, Diomedi-Camassei F, et al. Genetic homogeneity but IgG subclass-dependent clinical variability of alloimmune membranous nephropathy with anti-neutral endopeptidase antibodies. Kidney Int. 2015;87(3):602-9.

20 Prunotto M, Carnevali ML, Candiano G, Murtas C, Bruschi M, Corradini E, et al. Autoimmunity in membranous nephropathy targets aldose reductase and SOD2. J Am Soc Nephrol. 2010;21(3):507-19.

21 Han WW, Tang LJ, Kong XL, Yang H, Xu DM. Clinical significance of autoantibodies in the assessment and treatment of idiopathic membranous nephropathy. Exp Ther Med. 2019;17(3):1825-30.

22 Sethi S, Debiec H, Madden B, Charlesworth MC, Morelle J, Gross L, et al. Neural epidermal growth factor-like 1 protein (NELL-1) associated membranous nephropathy. Kidney Int. 2020;97(1):163-74.
23 Border WA, Ward HJ, Kamil ES, Cohen AH. Induction of membranous nephropathy in rabbits by administration of an exogenous cationic antigen. J Clin Invest. 1982;69(2):451-61.

24 Debiec H, Lefeu F, Kemper MJ, Niaudet P, Deschênes G, Remuzzi G, et al. Early-childhood membranous nephropathy due to cationic bovine serum albumin. N Engl J Med. 2011;364(22):2101-10.

$25 \mathrm{Ma} \mathrm{H}$, Sandor DG, Beck LH Jr. The role of complement in membranous nephropathy. Semin Nephrol. 2013;33(6):531-42.

26 Bally S, Debiec H, Ponard D, Dijoud F, Rendu J, Fauré J, et al. Phospholipase A2 receptorrelated membranous nephropathy and mannan-binding lectin deficiency. $\mathrm{J}$ Am Soc Nephrol. 2016;27(12):3539-44.

27 Chi JN, Lai TS, Wu CF, Fu TY, Chou YH, Chiu YL, et al. The relationship of anti-phospholipase $\mathrm{A} 2$ receptor antibody and $\mathrm{C} 5 \mathrm{a}$ complement with disease activity and short-term outcome in idiopathic membranous nephropathy. J Formos Med Assoc. 2019; 118(5):898-906.

28 Kerjaschki D. Pathomechanisms and molecular basis of membranous glomerulopathy. Lancet. 2004;364(9441):1194-6.

29 Stanescu HC, Arcos-Burgos M, Medlar A, Bockenhauer D, Kottgen A, Dragomirescu L, et al. Risk HLA-DQA1 and PLA(2)R1 alleles in idiopathic membranous nephropathy. $\mathrm{N}$ Engl J Med. 2011;364(7):616-26.

30 Bullich G, Ballarín J, Oliver A, Ayasreh N, Silva I, Santín S, et al. HLA-DQA1 and PLA2R1 polymorphisms and risk of idiopathic membranous nephropathy. Clin J Am Soc Nephrol. 2014;9(2):335-43.

31 Saeed M, Beggs ML, Walker PD, Larsen CP. PLA2R-associated membranous glomerulopathy is modulated by common variants in PLA2R1 and HLA-DQA1 genes. Genes Immun. 2014;15(8):556-61.

32 Kim S, Chin HJ, Na KY, Kim S, Oh J, Chung $\mathrm{W}$, et al. Single nucleotide polymorphisms in the phospholipase A2 receptor gene are associated with genetic susceptibility to idiopathic membranous nephropathy. Nephron Clin Pract. 2011;117(3):c253-8.

33 Ramachandran R, Kumar V, Kumar A, Yadav AK, Nada R, Kumar H, et al. PLA2R antibodies, glomerular PLA2R deposits and variations in PLA2R1 and HLA-DQA1 genes in primary membranous nephropathy in South Asians. Nephrol Dial Transplant. 2016; 31(9):1486-93.

34 Thiri M, Honda K, Kashiwase K, Mabuchi A, Suzuki H, Watanabe K, et al. High-density association mapping and interaction analysis of PLA2R1 and HLA regions with idiopathic membranous nephropathy in Japanese. Sci Rep. 2016;6:38189.

35 Kaga H, Komatsuda A, Omokawa A, Okuyama S, Mori K, Wakui H, et al. Analysis of PLA2R1 and HLA-DQA1 sequence variants in Japanese patients with idiopathic and secondary membranous nephropathy. Clin Exp Nephrol. 2018;22(2):275-82.
36 Lv J, Hou W, Zhou X, Liu G, Zhou F, Zhao N, et al. Interaction between PLA2R1 and HLADQA1 variants associates with anti-PLA2R antibodies and membranous nephropathy. J Am Soc Nephrol. 2013;24(8):1323-9.

37 Guang-yu Z, Yan-xia S, Li-xiang Z, Jing Y, Ying G, Min Y. Correlation of M-type phospholipase A2 receptor genetic polymorphism with idiopathic membranous nephropathy. J Chin J Nephrol. 2013;29(1):1-5.

38 Wang W, Fan S, Li G, Wang AY, Hong D, Zhong X, et al. Interaction between PLA2R1 and HLA-DQA1 Variants contributes to the increased genetic susceptibility to membranous nephropathy in Western China. Nephrology. 2018;24(9):919-25.

39 Le WB, Shi JS, Zhang T, Liu L, Qin HZ, Liang $S$, et al. HLA-DRB1*15:01 and HLADRB3*02:02 in PLA2R-related membranous nephropathy. J Am Soc Nephrol. 2017; 28(5):1642-50.

40 Wang HY, Cui Z, Xie LJ, Zhang LJ, Pei ZY, Chen FJ, et al. HLA class II alleles differing by a single amino acid associate with clinical phenotype and outcome in patients with primary membranous nephropathy. Kidney Int. 2018;94(5):974-82.

41 Chen Y-T, Chen C-H, Yen C-H, Chen S-Y, Tsai F-J. Association between MYH9 gene polymorphisms and membranous glomerulonephritis patients in Taiwan. ScienceAsia. 2013;39(6):625-30.

42 Remmers EF, Plenge RM, Lee AT, Graham RR, Hom G, Behrens TW, et al. STAT4 and the risk of rheumatoid arthritis and systemic lupus erythematosus. N Engl J Med. 2007; 357(10):977-86.

43 Taylor KE, Wong Q, Levine DM, McHugh C, Laurie C, Doheny K, et al. Genome-wide association analysis reveals genetic heterogeneity of Sjögren's syndrome according to ancestry. Arthritis Rheumatol. 2017;69(6):1294305 .

44 Rueda B, Broen J, Simeon C, Hesselstrand R, Diaz B, Suárez H, et al. The STAT4 gene influences the genetic predisposition to systemic sclerosis phenotype. Hum Mol Genet. 2009; 18(11):2071-7.

45 Zervou MI, Mamoulakis D, Panierakis C, Boumpas DT, Goulielmos GN. STAT4: a risk factor for type 1 diabetes? Hum Immunol. 2008;69(10):647-50.

46 Chen SY, Chen $\mathrm{CH}$, Huang YC, Chan CI, Hsieh YY, Yu MC, et al. Association of STAT4 polymorphisms with susceptibility to primary membranous glomerulonephritis and renal failure. Clin Chim Acta. 2011;412(2122):1899-904.

47 Chen SY, Chen CH, Huang YC, Chan CJ, Chen DC, Tsai FJ. Genetic susceptibility to idiopathic membranous nephropathy in high-prevalence Area, Taiwan. Biomedicine. 2014;4:9.

48 Honkanen E, von Willebrand E, Teppo AM, Törnroth T, Grönhagen-Riska C. Adhesion molecules and urinary tumor necrosis factoralpha in idiopathic membranous glomerulonephritis. Kidney Int. 1998;53(4):909-17. 
49 Neale TJ, Rüger BM, Macaulay H, Dunbar PR, Hasan Q, Bourke A, et al. Tumor necrosis factor-alpha is expressed by glomerular visceral epithelial cells in human membranous nephropathy. Am J Pathol. 1995;146(6):1444-54.

50 Bantis C, Heering PJ, Aker S, Siekierka M, Kuhr N, Grabensee B, et al. Tumor necrosis factor-alpha gene G-308A polymorphism is a risk factor for the development of membranous glomerulonephritis. Am J Nephrol. 2006;26(1):12-5.

51 Thibaudin D, Thibaudin L, Berthoux P, Mariat C, Filippis JP, Laurent B, et al. TNFA2 and $\mathrm{d} 2$ alleles of the tumor necrosis factor alpha gene polymorphism are associated with onset/occurrence of idiopathic membranous nephropathy. Kidney Int. 2007;71(5):431-7.

52 Zhanyun D, Yaping F, Lianglan S, Lan S, Feng $\mathrm{W}$, Haiquan L. Expression changes of nephrin and podocin in IMN, type $\mathrm{V}$ lupus nephritis and HBV associated membranous nephropathy. J China J Mod Medcine. 2009;19(15): 2381-5.

53 Lo WY, Chen SY, Wang HJ, Shih HC, Chen $\mathrm{CH}$, Tsai $\mathrm{CH}$, et al. Association between genetic polymorphisms of the NPHS1 gene and membranous glomerulonephritis in the Taiwanese population. Clin Chim Acta. 2010 411(9-10):714-8.

54 Chen CH, Shu KH, Wen MC, Chen KJ, Cheng $\mathrm{CH}$, Lian JD, et al. Impact of plasminogen activator inhibitor-1 gene polymorphisms on primary membranous nephropathy. Nephrol Dial Transplant. 2008;23(10):3166-73

55 Chen $\mathrm{CH}$, Chen SY, Shu KH, Wen MC, Cheng $\mathrm{CH}$, Wu MJ, et al. Urokinase gene 3'UTR T/C polymorphism is associated with malignancy and ESRD in idiopathic membranous nephropathy. Biomed Res Int. 2014; 2014:425095.

56 van de Logt AE, Fresquet M, Wetzels JF, Brenchley P. The anti-PLA2R antibody in membranous nephropathy: what we know and what remains a decade after its discovery. Kidney Int. 2019;96(6):1292-302.

57 Bobart SA, De Vriese AS, Pawar AS, Zand L, Sethi S, Giesen C, et al. Noninvasive diagnosis of primary membranous nephropathy using phospholipase A2 receptor antibodies. Kidney Int. 2019;95(2):429-38.

58 Du Y, Li J, He F, Lv Y, Liu W, Wu P, et al. The diagnosis accuracy of PLA2R-AB in the diagnosis of idiopathic membranous nephropathy: a meta-analysis. PLoS One. 2014;9(8): e104936.

59 Kaga H, Komatsuda A, Yamamoto S, Kikuchi T, Kamata M, Sato A, et al. Comparison of measurements of anti-PLA2R antibodies in Japanese patients with membranous nephropathy using in-house and commercial ELISA. Clin Exp Nephrol. 2019;23(4):465-73.

60 Pang L, Zhang AM, Li HX, Du JL, Jiao LL, Duan N, et al. Serum anti-PLA2R antibody and glomerular PLA2R deposition in Chinese patients with membranous nephropathy: a cross-sectional study. Medicine. 2017;96(24): e7218.
61 Debiec H, Ronco P. PLA2R autoantibodies and PLA2R glomerular deposits in membranous nephropathy. N Engl J Med. 2011; 364(7):689-90

62 Xie Q, Li Y, Xue J, Xiong Z, Wang L, Sun Z, et al. Renal phospholipase A2 receptor in hepatitis $\mathrm{B}$ virus-associated membranous nephropathy. Am J Nephrol. 2015;41(4-5):34553.

63 Ronco P, Debiec H. Anti-phospholipase A2 receptor antibodies and the pathogenesis of membranous nephropathy. Nephron Clin Pract. 2014;128(3-4):232-7.

64 Hofstra JM, Debiec H, Short CD, Pellé T, Kleta R, Mathieson PW, et al. Antiphospholipase A2 receptor antibody titer and subclass in idiopathic membranous nephropathy. J Am Soc Nephrol. 2012;23(10):1735-43.

65 Beck LH Jr, Fervenza FC, Beck DM, Bonegio RG, Malik FA, Erickson SB, et al. Rituximabinduced depletion of anti-PLA2R autoantibodies predicts response in membranous nephropathy. J Am Soc Nephrol. 2011;22(8): 1543-50.

$66 \mathrm{Wu}$ X, Liu L, Guo Y, Yang L. Clinical value of a serum anti-PLA2R antibody in the diagnosis and monitoring of primary membranous nephropathy in adults. Int J Nephrol Renovasc Dis. 2018;11:241-7.

67 Wu W, Shang J, Tao C, Wang S, Hu X, Zhang $S$, et al. The prognostic value of phospholipase A2 receptor autoantibodies on spontaneous remission for patients with idiopathic membranous nephropathy: A meta-analysis. Medicine. 2018;97(23):e11018.

68 Rodas LM, Matas-García A, Barros X, Blasco M, Viñas O, Llobell A, et al. Antiphospholipase 2 receptor antibody levels to predict complete spontaneous remission in primary membranous nephropathy. Clin Kidney J. 2019;12(1):36-41.

69 Cattran DC, Brenchley PE. Membranous nephropathy: integrating basic science into improved clinical management. Kidney Int. 2017;91(3):566-74.

70 De Vriese AS, Glassock RJ, Nath KA, Sethi S, Fervenza FC. A proposal for a serology-based approach to membranous nephropathy. J Am Soc Nephrol. 2017;28(2):421-30.

71 Ruggenenti P, Fervenza FC, Remuzzi G. Treatment of membranous nephropathy: time for a paradigm shift. Nat Rev Nephrol. 2017;13(9):563-79.

72 Hoxha E, Thiele I, Zahner G, Panzer U, Harendza S, Stahl RA. Phospholipase A2 receptor autoantibodies and clinical outcome in patients with primary membranous nephropathy. J Am Soc Nephrol. 2014;25(6): 1357-66.

73 Pourcine F, Dahan K, Mihout F, Cachanado M, Brocheriou I, Debiec H, et al. Prognostic value of PLA2R autoimmunity detected by measurement of anti-PLA2R antibodies combined with detection of PLA2R antigen in membranous nephropathy: a single-centre study over 14 years. PLoS One. 2017;12(3): e0173201.
74 Qu Z, Zhang MF, Cui Z, Wang J, Wang M, Zhang YM, et al. Antibodies against M-type phospholipase A2 receptor may predict treatment response and outcome in membranous nephropathy. Am J Nephrol. 2018;48(6):43846.

75 Song EJ, Jeong KH, Yang YA, Lim JH, Jung HY, Choi JY, et al. Erratum: anti-phospholipase A2 receptor antibody as a prognostic marker in patients with primary membranous nephropathy [Volume 37, Issue 3, September 2018, Pages 248-256]. Kidney Res Clin Pract. 2018;37(4):426-56.

76 Hofstra JM, Wetzels JFM. Should aspirin be used for primary prevention of thrombotic events in patients with membranous nephropathy? Kidney Int. 2016;89(5):981-3.

77 Zou H, Li Y, Xu G. Management of anticoagulation and antiplatelet therapy in patients with primary membranous nephropathy. BMC Nephrol. 2019;20(1):442.

78 Lionaki S, Derebail VK, Hogan SL, Barbour S, Lee T, Hladunewich M, et al. Venous thromboembolism in patients with membranous nephropathy. Clin J Am Soc Nephrol. 2012; 7(1):43-51.

79 Li SJ, Guo JZ, Zuo K, Zhang J, Wu Y, Zhou CS, et al. Thromboembolic complications in membranous nephropathy patients with nephrotic syndrome: a prospective study. Thromb Res. 2012;130(3):501-5.

80 Zou PM, Li H, Cai JF, Chen ZJ, Li C, Xu P, et al. A cohort study of incidences and risk factors for thromboembolic events in patients with idiopathic membranous nephropathy. Chin Med Sci J. 2018;33(2):91-9.

81 Lee T, Derebail VK, Kshirsagar AV, Chung Y, Fine JP, Mahoney S, et al. Patients with primary membranous nephropathy are at high risk of cardiovascular events. Kidney Int. 2016;89(5):1111-8.

82 Radice A, Pieruzzi F, Trezzi B, Ghiggeri G, Napodano P, D'Amico M, et al. Diagnostic specificity of autoantibodies to M-type phospholipase A2 receptor (PLA2R) in differentiating idiopathic membranous nephropathy (IMN) from secondary forms and other glomerular diseases. J Nephrol. 2018;31(2):2718.

83 Garcia-Vives E, Solé C, Moliné T, AlvarezRios AM, Vidal M, Agraz I, et al. Antibodies to M-type phospholipase A2 receptor (PLA2R) in membranous lupus nephritis. Lupus. 2019;28(3):396-405.

84 Sethi S, Madden BJ, Debiec H, Charlesworth MC, Gross L, Ravindran A, et al. Exostosin 1/ exostosin 2-associated membranous nephropathy. J Am Soc Nephrol. 2019;30(6): 1123-36.

85 Tian CX, Li L, Qiu P, Qiu YR. Association of SNPs in PLA2R1 with idiopathic and secondary membranous nephropathy in two Chinese cohorts. Br J Biomed Sci. 2019;77(1):248 . 